\title{
ON THE CONNECTEDNESS OF THE BRANCH LOCI OF NON-ORIENTABLE UNBORDERED KLEIN SURFACES OF LOW GENUS
}

\author{
E. BUJALANCE \\ Departamento de Matemáticas Fundamentales, UNED, Paseo \\ Senda del Rey 9, 28040-Madrid, Spain \\ e-mail: ebujalance@mat.uned.es \\ J. J. ETAYO \\ Departamento de Álgebra, Facultad de Matemáticas, \\ Universidad Complutense, 28040-Madrid, Spain \\ e-mail:jetayo@mat.ucm.es \\ E. MARTÍNEZ \\ Departamento de Matemáticas Fundamentales, UNED, \\ Paseo Senda del Rey 9, 28040-Madrid, Spain \\ e-mail: emartinez@mat.uned.es \\ and B. SZEPIETOWSKI \\ Institute of Mathematics, University of Gdańsk. Ul. Wita \\ Stwosza 57, 80-952 Gdańsk, Poland \\ e-mail: blaszep@mat.ug.edu.pl
}

(Received 20 November 2012; revised 10 April 2014; accepted 15 May 2014)

\begin{abstract}
This paper is devoted to determine the connectedness of the branch loci of the moduli space of non-orientable unbordered Klein surfaces. We obtain a result similar to Nielsen's in order to determine topological conjugacy of automorphisms of prime order on such surfaces. Using this result we prove that the branch locus is connected for surfaces of topological genus 4 and 5 .
\end{abstract}

2010 Mathematics Subject Classification. Primary 57M60; Secondary 20F05, 20H10, 30F50.

1. Introduction. A non-orientable unbordered surface is called a Klein surface if it is compact and endowed with a dianalytic atlas, see [1]. Non-orientable unbordered Klein surfaces are also called non-orientable Riemann surfaces and they correspond to the purely imaginary real algebraic curves.

The study of the moduli space of both Riemann and Klein surfaces is a classic problem. The subspace of surfaces with non-trivial automorphisms is called the branch locus of the moduli space. In the case of Riemann surfaces this subspace was studied by Harvey in [14] and Broughton in [5]. Its connectedness was studied in several papers, for example in $[\mathbf{3}, \mathbf{1 0}, \mathbf{1 3}, \mathbf{1 5}]$. The main result is that the real locus of the moduli space of Riemann surfaces is connected (see [11, 12, 20]), while the branch locus of the moduli space of Riemann surfaces of genus $g$ is connected only when $g$ is $3,4,7,13,17,19$ or 59 (see $[2,4])$. 
In this paper we determine the connectedness of the branch locus for nonorientable unbordered Klein surfaces of topological genera 4 and 5 . We do not consider surfaces of genus 3 because they are hyperelliptic and it is already known that the branch locus is connected. In order to make that study in genus 4 and 5 we start from the results on the moduli space and the branch locus of Klein surfaces obtained by Macbeath and Singerman in [17], Natanzon in [18] and Seppälä in [20].

In Section 3, we establish an analog of Nielsen's result on the parameters determining an automorphism of prime order on a given compact Riemann surface up to topological conjugation. Here we are concerned with non-orientable unbordered Klein surfaces. Using this result we obtain in the remaining sections that the branch loci of these surfaces are connected for genus 4 and 5. Before this, in Section 2, we give the preliminaries about NEC groups and Klein surfaces, and explain the technique used to check that the branch locus is connected. In the last sections we consider the surfaces with genera 4 and 5.

\section{Preliminaries.}

2.1. NEC groups. An NEC group $\Gamma$ is a discrete subgroup of isometries of the hyperbolic plane $\mathcal{H}$, including orientation-reversing elements, with compact quotient $S=\mathcal{H} / \Gamma$. Each NEC group $\Gamma$ has associated a signature [16]:

$$
\sigma(\Gamma)=\left(g, \pm,\left[m_{1}, \ldots, m_{r}\right],\left\{\left(n_{i, 1}, \ldots, n_{i, s_{i}}\right), i=1, \ldots, k\right\}\right)
$$

where $g, k, r, m_{i}, n_{i, j}$ are integers satisfying $g, k, r \geq 0, m_{i} \geq 2, n_{i, j} \geq 2$. The number $g$ is the topological genus of $S$. The sign determines the orientability of $S$. The numbers $m_{i}$ are the proper periods. The brackets $\left(n_{i, 1}, \ldots, n_{i, s_{i}}\right)$ are the period-cycles. The number $k$ of period-cycles is equal to the number of boundary components of $S$. Numbers $n_{i, j}$ are the periods of the period-cycle $\left(n_{i, 1}, \ldots, n_{i, s_{i}}\right)$, also called link-periods. We will denote by [-] and (-) the cases when $r=0$ and $s_{i}=0$ respectively. Also, we abbreviate by $\left[(m)^{r}\right]$ and $\left\{(-)^{k}\right\}$ the expressions $[m, . r ., m]$ and $\{(-), . k .,(-)\}$ respectively.

The signature determines a presentation [22] of $\Gamma$ by means of generators $x_{i}$ $(i=1, \ldots, r) ; e_{i}(i=1, \ldots, k) ; c_{i, j}\left(i=1, \ldots, k ; j=0, \ldots, s_{i}\right)$; and $a_{i}, b_{i}(i=1, \ldots, g)$ if $\sigma$ has sign ' + ' and $d_{i}(i=1, \ldots, g)$ if $\sigma$ has sign '-'.

These generators satisfy the following relations: $x_{i}^{m_{i}}=1(i=1, \ldots, r) ; c_{i, j-1}^{2}=$ $c_{i, j}^{2}=\left(c_{i, j-1} c_{i, j}\right)^{n_{i, j}}=1 \quad\left(i=1, \ldots, k, j=1, \ldots, s_{i}\right) ; \quad e_{i}^{-1} c_{i, 0} e_{i} c_{i, s_{i}}=1 \quad(i=1, \ldots, k) ;$ $\prod_{i=1}^{r} x_{i} \prod_{i=1}^{k} e_{i} \prod_{i=1}^{g}\left(a_{i} b_{i} a_{i}^{-1} b_{i}^{-1}\right)=1$ if $\sigma$ has sign ' + '; and $\prod_{i=1}^{r} x_{i} \prod_{i=1}^{k} e_{i} \prod_{i=1}^{g} d_{i}^{2}=1$ if $\sigma$ has sign ' - '.

The corresponding final relation is called the long relation. The isometries $x_{i}$ are elliptic, $e_{i}, a_{i}, b_{i}$ are hyperbolic, $c_{i, j}$ are reflections and $d_{i}$ are glide reflections.

Every NEC group $\Gamma$ with signature (1) has associated a fundamental region whose area $\mu(\Gamma)$, called the area of the group, is:

$$
\mu(\Gamma)=2 \pi\left(\eta g+k-2+\sum_{i=1}^{r}\left(1-\frac{1}{m_{i}}\right)+\frac{1}{2} \sum_{i=1}^{k} \sum_{j=1}^{s_{i}}\left(1-\frac{1}{n_{i, j}}\right)\right),
$$


with $\eta=2$ or 1 depending on whether the sign in the signature is ' + ' or ' - '. An NEC group with signature (1) actually exists if and only if the right-hand side of (2) is greater than 0 .

If $\Gamma$ is a subgroup of an NEC group $\Lambda$ of finite index $N$, then also $\Gamma$ is an NEC group and the following Riemann-Hurwitz formula holds:

$$
\mu(\Gamma)=N \mu(\Lambda)
$$

Let $S$ be a non-orientable Klein surface without boundary of topological genus $g \geq 3$. Then, by [19], there exists an NEC group $\Gamma_{g}$ with signature:

$$
\sigma\left(\Gamma_{g}\right)=(g,-,[-],\{-\})
$$

such that $S=\mathcal{H} / \Gamma_{g}$.

2.2. Teichmüller and moduli spaces. Let $\mathcal{G}$ be the group of isometries of the hyperbolic plane $\mathcal{H}$, including reversing-orientation elements. Given an NEC group $\Gamma$, we denote by $R(\Gamma)$ the set of monomorphisms $r: \Gamma \rightarrow \mathcal{G}$ such that $r(\Gamma)$ is discrete and $\mathcal{H} / \Gamma$ is compact.

Two elements $r_{1}, r_{2} \in R(\Gamma)$ are said to be equivalent if there exists $g \in \mathcal{G}$ such that $r_{1}(\gamma)=g r_{2}(\gamma) g^{-1}$ for each $\gamma \in \Gamma$. The quotient space $T(\Gamma)$ is the Teichmüller space of $\Gamma$.

When $\Gamma$ is a Fuchsian group with signature $\left(g,+,\left[m_{1}, \ldots, m_{r}\right]\right)$, it is known as that the dimension of $T(\Gamma), d(T(\Gamma))$ is $6(g-1)+2 r$. Let now $\Gamma$ be a proper NEC group and call $\Gamma^{+}$its canonical Fuchsian subgroup. Singerman proved in [21] that $d(T(\Gamma))=$ $\frac{1}{2} d\left(T\left(\Gamma^{+}\right)\right)$. The Teichmüller modular group of $\Gamma, \operatorname{Mod}(\Gamma)$, is the quotient group $\operatorname{Aut}(\Gamma) / \operatorname{Inn}(\Gamma)$, where $\operatorname{Aut}(\Gamma)$ and $\operatorname{Inn}(\Gamma)$ denote the full group of automorphisms and the subgroup of inner automorphisms of $\Gamma$ respectively [17]. The moduli space of $\Gamma$ is the quotient $M(\Gamma)=T(\Gamma) / \operatorname{Mod}(\Gamma)$. For the action of the $\operatorname{Mod}(\Gamma)$ on the Teichmüller space, we refer the reader to Section 9 in [17].

Let $K$ be an NEC subgroup of $\Gamma$ of finite index. Then following the notation from Harvey in [14], let $I(K, \Gamma, H)$ denote the set of all injections $i: K \hookrightarrow \Gamma$ with $\Gamma / i(K) \approx H$. Every such $i$ is determined up to automorphisms of $K$ by a surjection of $\Gamma$ onto $H$. Let $\Phi(K, \Gamma, H)$ be the family of all equivalence classes of surjections $\varphi: \Gamma \rightarrow H$ with $\operatorname{ker}(\varphi) \approx K$, modulo the actions of $\operatorname{Aut}(\Gamma)$ and $\operatorname{Aut}(H)$. Note that $\Phi$ is finite since $\Gamma$ is finitely generated.

Given $i: K \hookrightarrow \Gamma$, denote by $T(\hat{l})$ the image of the associated isometric embedding $T(\Gamma) \rightarrow T(K)$ (see [17]). Let $i_{\varphi}$ be any injection corresponding to a class $[\varphi] \in$ $\Phi(K, \Gamma, H), i_{\varphi}: K \rightarrow \operatorname{ker}(\varphi)$. We denote by $\Lambda(K, \Gamma, H)$ the set $\{[r] \in T(K):[r] \in$ $T(\hat{\imath}), i \in I(K, \Gamma, H)\}$. Then,

$$
\Lambda(K, \Gamma, H)=\bigcup_{\alpha \in \operatorname{Mod}(K)} \alpha\left(\bigcup_{[\varphi] \in \Phi(K, \Gamma, H)} T\left(\hat{\imath}_{\varphi}\right)\right) .
$$

Let $\Gamma_{g}$ be an NEC group with signature $(g,-,[-],\{-\})$. Then $T\left(\Gamma_{g}\right)$ is the Teichmüller space of compact non-orientable Klein surfaces without boundary of topological genus $g$. We denote by $\Lambda_{g}$ the subspace of $T\left(\Gamma_{g}\right)$ corresponding to those surfaces with non-trivial automorphisms. Then, by [17], $\Lambda_{g}$ consists of those points 
which are fixed by some element of $\operatorname{Mod}\left(\Gamma_{g}\right)$, which must have finite order. Let $n$ range over all possible orders of elements of $\operatorname{Mod}\left(\Gamma_{g}\right)$, in fact, $2 \leq n \leq 2 g$ if $g$ is odd, and $2 \leq n \leq 2(g-1)$ if $g$ is even, see [6]. For each $n$, let $\mathcal{F}_{n, g}$ denote the set of isomorphism classes of NEC groups $\Gamma$ with non-empty $\Phi\left(\Gamma_{g}, \Gamma, C_{n}\right)$, where $C_{n}$ is the cyclic group of order $n$. Then,

$$
\Lambda_{g}=\bigcup_{n} \bigcup_{\Gamma \in \mathcal{F}_{n, g}} \Lambda\left(\Gamma_{g}, \Gamma, C_{n}\right) .
$$

Hence, $\Lambda_{g}$ is a countable union of submanifolds of $T\left(\Gamma_{g}\right)$. We call $B_{g}=$ $\Lambda_{g} / \operatorname{Mod}\left(\Gamma_{g}\right)$. Each set $\Lambda\left(\Gamma_{g}, \Gamma, C_{n}\right)$ is mapped into itself by $\operatorname{Mod}\left(\Gamma_{g}\right)$, and the quotient of this set is made up of connected subsets in a one-to-one correspondence with classes $[\varphi] \in \Phi\left(\Gamma_{g}, \Gamma, C_{n}\right)$.

Let $\theta_{i}: \Gamma_{i} \rightarrow G, i=1,2$ be two epimorphisms with $\operatorname{ker} \theta_{i} \approx \Gamma_{g}$, where $G$ is a finite group and $\Gamma_{i}$ are NEC groups. We say that $\theta_{1}$ and $\theta_{2}$ are topologically conjugate if and only if there exist an isomorphism $\psi: \Gamma_{1} \rightarrow \Gamma_{2}$ and $\alpha \in \operatorname{Aut}(G)$ such that $\alpha \theta_{1}=\theta_{2} \psi$.

Given two primes $p$ and $q$ and two NEC groups $\Gamma^{\prime}$ and $\Gamma^{\prime \prime}$, consider two epimorphisms $\theta^{\prime}: \Gamma^{\prime} \rightarrow C_{p}$ and $\theta^{\prime \prime}: \Gamma^{\prime} \rightarrow C_{q}$ with $\operatorname{ker}\left(\theta^{\prime}\right) \approx \operatorname{ker}\left(\theta^{\prime \prime}\right) \approx \Gamma_{g}$. Then we say that $\theta^{\prime}$ and $\theta^{\prime \prime}$ are connected if there exist an NEC group $\Gamma^{*}$ and an epimorphism $\theta^{*}: \Gamma^{*} \rightarrow G$, where $G$ is a group of order $p q$, and there exist two subgroups of $G, G^{\prime}$ and $G^{\prime \prime}$, of orders $p$ and $q$, satisfying

$$
\begin{aligned}
& \theta^{*^{-1}}\left(G^{\prime}\right) \approx \Gamma^{\prime}, \\
& \theta^{*^{-1}}\left(G^{\prime \prime}\right) \approx \Gamma^{\prime \prime}, \\
& \left.\theta^{*}\right|_{\theta^{*-1}\left(G^{\prime}\right)} \text { is topologically conjugate to } \theta^{\prime}, \\
& \left.\theta^{*}\right|_{\theta^{*-1}\left(G^{\prime \prime}\right)} \text { is topologically conjugate to } \theta^{\prime \prime} .
\end{aligned}
$$

Since each non-trivial group $G$ contains a subgroup of prime order, for proving that $B_{g}$ is connected, it suffices to prove that for each primes $p, q$, each two epimorphisms $\theta^{\prime}$, $\theta^{\prime \prime}$, there exist a sequence of primes $p_{i}$, NEC groups $\Gamma_{i}$ and epimorphisms $\theta_{i}: \Gamma_{i} \rightarrow C_{p_{i}}$, $1 \leq i \leq k$, such that $\theta_{1}=\theta^{\prime}, \theta_{k}=\theta^{\prime \prime}$ and $\theta_{i}$ is connected to $\theta_{i+1}$ for $i=1, \ldots, k-1$. This will be done in Sections 4 and 5 for primes 2 and 3 (genus 4 ) and for 2, 3 and 5 (genus 5). For a similar argument, see for example [2] or [3].

3. Equivalence. Suppose that $\theta_{i}: \Gamma \rightarrow C_{p}, i=1,2$ are two epimorphisms, where $\Gamma$ is an NEC group, $p$ is prime and $\operatorname{ker} \theta_{i}$ is isomorphic to the fundamental group of a non-orientable surface. In this section, we give necessary and sufficient conditions for $\theta_{1}$ and $\theta_{2}$ to be topologically conjugate.

We list the automorphisms of $\Gamma$ which will be used. Most of these are from papers $[7,14]$. If the sign of the signature of $\Gamma$ is ' + ', then we are going to use the following automorphisms of $\Gamma$ :

$\omega$ defined by $\omega\left(a_{1}\right)=a_{1} b_{1}$ and the identity on the remaining canonical generators.

$\xi$ defined by $\xi\left(a_{1}\right)=a_{1} b_{1}, \xi\left(b_{1}\right)=a_{1}^{-1}$ and the identity on the remaining canonical generators.

$v_{j}$ defined by $v_{j}\left(a_{j}\right)=a_{j+1}, v_{j}\left(b_{j}\right)=b_{j+1}, v_{j}\left(a_{j+1}\right)=c_{j+1}^{-1} a_{j} c_{j+1}, v_{j}\left(b_{j+1}\right)=c_{j+1}^{-1} b_{j} c_{j+1}$, where $c_{j+1}=\left[a_{j+1}, b_{j+1}\right]$ and the identity on the remaining canonical generators.

$\mu$ defined by $\mu\left(a_{1}\right)=a_{2} a_{1}, \mu\left(a_{2}\right)=b_{1} a_{2} b_{1}^{-1}, \mu\left(b_{1}\right)=b_{1}, \mu\left(b_{2}\right)=a_{2} b_{2} a_{2}^{-1} b_{1}^{-1}$ and $\mu(y)=a_{2} y a_{2}^{-1}$ for every canonical generator $y$ different from $a_{1}, b_{1}, a_{2}, b_{2}$. 
$\sigma$ defined by $\sigma\left(x_{r}\right)=E a_{1}^{-1} E^{-1} x_{r} E a_{1} E^{-1}, \sigma\left(a_{1}\right)=\left[a_{1}^{-1}, E^{-1} x_{r}^{-1} E\right] a_{1}, \sigma\left(b_{1}\right)=$ $b_{1} a_{1}^{-1} E^{-1} x_{r} E a_{1}$, where $E=e_{1} \cdots e_{k}$ and the identity on the remaining canonical generators.

$\pi$ defined by $\pi\left(e_{k}\right)=a_{1}^{-1} e_{k} a_{1}, \pi\left(c_{k, i}\right)=a_{1}^{-1} c_{k, i} a_{1}, \pi\left(a_{1}\right)=\left[a_{1}^{-1}, e_{k}^{-1}\right] a_{1}, \pi\left(b_{1}\right)=$ $b_{1} a_{1}^{-1} e_{k} a_{1}$ and the identity on the remaining canonical generators.

If the sign in the signature of $\Gamma$ is '-', then we are going to use the following automorphisms.

$\alpha_{j}$ defined by $\alpha_{j}\left(d_{j}\right)=d_{j}^{2} d_{j+1} d_{j}^{-2}, \alpha_{j}\left(d_{j+1}\right)=d_{j}$ and the identity on the remaining canonical generators.

$\beta_{j}$ defined by $\beta_{j}\left(d_{j}\right)=d_{j} d_{j+1}^{-1} d_{j}^{-1}, \beta_{j}\left(d_{j+1}\right)=d_{j} d_{j+1}^{2}$ and the identity on the remaining canonical generators.

$\gamma$ defined by $\gamma\left(d_{1}\right)=E^{-1} x_{r} E d_{1}, \gamma\left(x_{r}\right)=x_{r} E d_{1} E^{-1} x_{r}^{-1} E d_{1}^{-1} E^{-1} x_{r}^{-1}$, where $E=$ $e_{1} \cdots e_{k}$ and the identity on the remaining canonical generators.

$\varepsilon$ defined for $s_{k}=0$ by $\varepsilon\left(d_{1}\right)=e_{k} d_{1}, \quad \varepsilon\left(e_{k}\right)=e_{k} d_{1} e_{k}^{-1} d_{1}^{-1} e_{k}^{-1}, \quad \varepsilon\left(c_{k, 0}\right)=$ $e_{k} d_{1} c_{k, 0} d_{1}^{-1} e_{k}^{-1}$ and the identity on the remaining canonical generators.

$\eta$ defined for $g \geq 4$ by

$$
\begin{array}{ll}
\eta\left(d_{1}\right)=d_{1} d_{2} d_{3} d_{4} d_{1}, & \eta\left(d_{2}\right)=\left(d_{3} d_{4} d_{1}\right)^{-1}, \\
\eta\left(d_{3}\right)=d_{3} d_{4} d_{1} d_{2} d_{3}, & \eta\left(d_{4}\right)=\left(d_{1} d_{2} d_{3}\right)^{-1},
\end{array}
$$

and $\eta(y)=\left(d_{1} d_{2} d_{3} d_{4}\right) y\left(d_{1} d_{2} d_{3} d_{4}\right)^{-1}$ for every canonical generator different from $d_{1}, d_{2}, d_{3}, d_{4}$.

Regardless of the sign in the signature, we are also going to use the following automorphisms.

$\rho_{i}$ defined by $\rho_{i}\left(x_{i}\right)=x_{i} x_{i+1} x_{i}^{-1}, \rho_{i}\left(x_{i+1}\right)=x_{i}$ and the identity on the remaining canonical generators.

$\lambda_{i}$ defined for $s_{i}=s_{i+1}=0$ by $\lambda_{i}\left(e_{i}\right)=e_{i} e_{i+1} e_{i}^{-1}, \lambda_{i}\left(e_{i+1}\right)=e_{i}, \lambda_{i}\left(c_{i, 0}\right)=e_{i} c_{i+1,0} e_{i}^{-1}$, $\lambda_{i}\left(c_{i+1,0}\right)=c_{i, 0}$ and the identity on the remaining canonical generators.

THEOREM 1. Suppose that $p$ is an odd prime, $\Gamma$ is an NEC group of signature $\left(g ;-;\left[(p)^{r}\right] ;\{-\}\right)$ and $\theta_{i}: \Gamma \rightarrow C_{p}$ for $i=1,2$ are two epimorphisms with $\operatorname{ker} \theta_{i}$ isomorphic to the fundamental group of a non-orientable surface. Then $\theta_{1}$ and $\theta_{2}$ are topologically conjugate if and only if $\left(\theta_{2}\left(x_{1}\right), \ldots, \theta_{2}\left(x_{r}\right)\right)$ is a permutation of $\left(\left(\theta_{1}\left(x_{1}\right)\right)^{\varepsilon_{1} a}, \ldots,\left(\theta_{1}\left(x_{r}\right)\right)^{\varepsilon_{r} a}\right)$ for some $a \in\{1, \ldots, p-1\}$ and $\varepsilon_{j} \in\{-1,1\}, j=1, \ldots, r$.

Proof. The 'only if' part follows from the fact that every automorphism of $\Gamma$ maps $x_{i}$ to a conjugate of some $x_{j}$ or $x_{j}^{-1}$, see [16]. We are going to prove the 'if' part.

Suppose that $r>0$. By using the automorphisms $\rho_{i}, \gamma$ we may obtain a new system of canonical generators $x_{1}^{\prime}, \ldots, x_{r}^{\prime}, d_{1}^{\prime}, \ldots, d_{g}^{\prime}$ for which $\theta_{2}\left(x_{i}^{\prime}\right)=\left(\theta_{1}\left(x_{i}^{\prime}\right)\right)^{a}$ for $i=1, \ldots, r$. By using the automorphisms $\alpha_{j}$ we can permute the generators $d_{j}^{\prime}$ so that $\theta_{1}\left(d_{j}^{\prime}\right)=1$ for $j \geq m$ and $\theta_{1}\left(d_{j}^{\prime}\right) \neq 1$ for $j<m$. Suppose that $m>2$. There exists $b$ such that $\theta_{1}\left(d_{1}^{\prime}\right)\left(\theta\left(x_{r}^{\prime}\right)\right)^{b}=1$. With the automorphism $\left(\gamma \alpha_{1} \gamma \alpha_{1}\right)^{b}$ we obtain a new system of generators $x_{1}^{\prime \prime}, \ldots, x_{r}^{\prime \prime}, d_{1}^{\prime \prime}, \ldots, d_{g}^{\prime \prime}$ such that $\theta_{1}\left(x_{i}^{\prime \prime}\right)=\theta_{1}\left(x_{i}^{\prime}\right)$ for $i=1, \ldots, r, \theta_{1}\left(d_{j}^{\prime \prime}\right)=1$ for $j \geq m$ and $\theta_{1}\left(d_{1}^{\prime \prime}\right)=1$. Reordering the $d_{j}^{\prime \prime}$, we have a new system of generators such that $\theta_{1}\left(d_{j}^{\prime \prime}\right)=1$ for $j \geq m-1$. By repeating this process we can obtain a system of generators, where $\theta_{1}\left(d_{j}^{\prime \prime}\right)=1$ for $j>1$ and $\theta_{1}\left(d_{1}^{\prime \prime}\right)$ is determined by

$$
\theta_{1}\left(x_{1}^{\prime \prime} \cdots x_{r}^{\prime \prime}\left(d_{1}^{\prime \prime}\right)^{2} \cdots\left(d_{g}^{\prime \prime}\right)^{2}\right)=1
$$


Analogously we can find a system of generators $x_{1}^{\prime \prime \prime}, \ldots, x_{r}^{\prime \prime \prime}, d_{1}^{\prime \prime \prime}, \ldots, x_{g}^{\prime \prime \prime}$ such that $\theta_{2}\left(x_{i}^{\prime \prime \prime}\right)=\theta_{2}\left(x_{i}^{\prime}\right)$ for $i=1, \ldots, r$ and $\theta_{2}\left(d_{j}^{\prime \prime \prime}\right)=1$ for $j>1$. It follows that there exists $\psi \in \operatorname{Aut}(\Gamma)$ and $\alpha \in \operatorname{Aut}\left(C_{p}\right)$ such that $\theta_{2} \psi=\alpha \theta_{1}$.

Suppose that $r=0$. By using the automorphisms $\alpha_{j}$ and $\beta_{j}$ we can find a new system of generators $d_{1}^{\prime}, \ldots, d_{g}^{\prime}$ such that $\theta_{1}\left(d_{i}^{\prime}\right)=X$ for $i>1$, where $X \neq 1$, and $\theta_{1}\left(d_{1}^{\prime}\right)$ is determined by the long relation ( $\mathrm{cf}$ [7, proof of Theorem 3]). Since we can do the same for $\theta_{2}$, there exist $\psi \in \operatorname{Aut}(\Gamma)$ and $\alpha \in \operatorname{Aut}\left(C_{p}\right)$ such that $\theta_{2} \psi=\alpha \theta_{1}$.

The next result can be obtained from [8]. However, we give here a direct proof.

THEOREM 2. Suppose that $\Gamma$ is an NEC group of signature $\left(g ; \pm ;\left[(2)^{r}\right] ;\left\{(-)^{k}\right\}\right)$ and $\theta_{i}: \Gamma \rightarrow C_{2}$ for $i=1,2$ are two epimorphisms with $\operatorname{ker} \theta_{i}$ isomorphic to the fundamental group of a non-orientable surface. Let $X$ be the generator of $C_{2}$ and

$$
n_{i}=\#\left\{j \in\{1, \ldots, k\} \mid \theta_{i}\left(e_{j}\right)=X\right\} \quad \text { for } i=1,2 .
$$

Then $\theta_{1}$ and $\theta_{2}$ are topologically conjugate if and only if

(1) $n_{1}=n_{2}$, and if $r=n_{1}=n_{2}=0$ and the sign is ' - ' then

(2) $\theta_{1}\left(d_{1} \cdots d_{g}\right)=\theta_{2}\left(d_{1} \cdots d_{g}\right)$ and

(3) $\theta_{1}\left(d_{1}\right)=\cdots=\theta_{1}\left(d_{g}\right)=1$ if and only if $\theta_{2}\left(d_{1}\right)=\cdots=\theta_{2}\left(d_{g}\right)=1$.

Proof. Let $\theta: \Gamma \rightarrow C_{2}$ be an epimorphism with $\operatorname{ker} \theta$ isomorphic to the fundamental group of a non-orientable surface, and $n=\#\left\{j \in\{1, \ldots, k\} \mid \theta_{i}\left(e_{j}\right)=X\right\}$.

Case 1. $r+n>0$.

We are going to show that in this case $\theta$ is determined by $n$ up to an automorphism of $\Gamma$. Since $\operatorname{ker} \theta$ is torsion-free, we have $\theta\left(x_{i}\right)=X$ for $i=1, \ldots, r$ and $\theta\left(c_{j, 0}\right)=X$ for $j=1, \ldots, k$. The automorphisms $\lambda_{i}$ permute the boundary generators $e_{i}$ up to conjugation, so we may assume that $\theta\left(e_{i}\right)=1$ for $i \leq k-n$ and $\theta\left(e_{i}\right)=X$ for $i>k-n$.

Subcase 1(a) The sign of the signature is ' - '. By using the automorphisms $\alpha_{j}$ and $\varepsilon$ if $n>0$ or $\gamma$ if $r>0$, we can obtain a new system of canonical generators such that $\theta\left(d_{i}\right)=1$ for $i=1, \ldots, g$.

Subcase 1(b) The sign of the signature is ' + '. Suppose that $\theta\left(a_{i}\right)=\theta\left(b_{i}\right)=1$ for $i=1, \ldots, g$. Then by using the automorphism $\pi$ if $n>0$ or $\sigma$ if $r>0$, we obtain a new system of canonical generators such that $\theta\left(b_{1}\right)=X$. Therefore, we may assume that $\theta\left(a_{i}\right)=X$ or $\theta\left(b_{i}\right)=X$ for some $i$ and by the automorphisms $v_{i}$ we may assume that $i=1$. There exists $\psi \in \operatorname{Aut}(\Gamma)$ such that $\theta\left(\psi\left(a_{1}\right)\right)=\theta\left(\psi\left(b_{1}\right)\right)=X$ and $\theta(\psi(y))=\theta(y)$ for every canonical generator $y$ different from $a_{1}$ and $b_{1}$. Indeed, if $\theta\left(a_{1}\right)=1$ and $\theta\left(b_{1}\right)=X$, then $\psi=\omega$, whereas if $\theta\left(a_{1}\right)=X$ and $\theta\left(b_{1}\right)=1$, then $\psi=\xi$. Therefore, we may assume that $\theta\left(a_{i}\right)=\theta\left(b_{i}\right)=X$ for $i \leq m$ and $\theta\left(a_{i}\right)=\theta\left(b_{i}\right)=1$ for $i>m$ for some $m>0$. Suppose that $m<g$. Then after reordering the generators by the automorphisms $v_{i}$ we may assume that $\theta\left(a_{1}\right)=\theta\left(b_{1}\right)=1$ and $\theta\left(a_{2}\right)=\theta\left(b_{2}\right)=X$. Now for $\psi=\xi \mu$ we have $\theta\left(\psi\left(a_{1}\right)\right)=\theta\left(\psi\left(b_{1}\right)\right)=X$ and $\theta(\psi(y))=\theta(y)$ for every canonical generator $y$ different from $a_{1}$ and $b_{1}$. Repeating this process we arrive at a new system of canonical generators such that $\theta\left(a_{i}\right)=\theta\left(b_{i}\right)=X$ for $i=1, \ldots, g$.

Case 2. $r=n=0$.

Subcase 2(a) The sign of the signature is ' + '. Since $\mathcal{H} / \operatorname{ker} \theta$ is non-orientable, $\theta\left(a_{i}\right)=X$ or $\theta\left(b_{i}\right)=X$ for some $i$. Proceeding as in subcase 1(b), we can find a system of canonical generators such that $\theta\left(a_{i}\right)=\theta\left(b_{i}\right)=X$ for $i=1, \ldots, g$. 
Subcase 2(b) The sign of the signature is '-'. Let

$$
m=\#\left\{j \in\{1, \ldots, g\} \mid \theta_{1}\left(d_{j}\right)=X\right\} .
$$

The condition $m=0$ clearly determines $\theta$. We are going to show that if $m>0$, then $\theta$ is determined up to an automorphism of $\Gamma$ by $m \bmod 2$. Since $\mathcal{H} / \operatorname{ker} \theta$ is nonorientable, $m<g$. Suppose that $m \geq 3$. Reordering the $d_{i}$ s we may assume that $\theta\left(d_{1}\right)=$ $\theta\left(d_{2}\right)=\theta\left(d_{3}\right)=X$ and $\theta\left(d_{4}\right)=1$. Then $\theta\left(\eta\left(d_{1}\right)\right)=\theta\left(\eta\left(d_{2}\right)\right)=\theta\left(\eta\left(d_{3}\right)\right)=1, \theta\left(\eta\left(d_{4}\right)\right)=$ $X$ and $\theta(\eta(y))=\theta(y)$ for every canonical generator $y$ different from $d_{1}, d_{2}, d_{3}, d_{4}$. Repeating this process we can find a system of canonical generators such that if $m$ was odd then $\theta\left(d_{1}\right)=X$ and $\theta\left(d_{i}\right)=1$ for $i \geq 2$, and if $m$ was even then $\theta\left(d_{1}\right)=\theta\left(d_{2}\right)=X$ and $\theta\left(d_{i}\right)=1$ for $i \geq 3$.

We have proved that the conditions (1)-(3) are sufficient for $\theta_{1}$ and $\theta_{2}$ to be topologically conjugate. Now we are going to prove that they are also necessary. The necessity of condition (1) follows from the fact that every automorphism of $\Gamma$ maps $e_{i}$ to a conjugate of some $e_{j}$ or $e_{j}^{-1}$, see [16]. Suppose that the sign is ' ${ }^{-}{ }^{\prime}, r=n_{1}=n_{2}=0$ and $\theta_{2}=\theta_{1} \psi$ for some $\psi \in \operatorname{Aut}(\Gamma)$. Let $K$ be the smallest normal subgroup of $\Gamma$ containing $e_{1}, \ldots, e_{k}$ and the commutator subgroup $[\Gamma, \Gamma]$. Let $H=\Gamma / K$ and $\overline{c_{i}}, \overline{d_{j}} \in H$ be the projections of $c_{i, 0}, d_{j}$ respectively. As a $\mathbb{Z}$-module, $H$ has the presentation

$$
\left\langle\overline{c_{1}}, \ldots, \overline{c_{k}}, \overline{d_{1}}, \ldots, \overline{d_{g}} \mid 2 \overline{c_{1}}=\cdots=2 \overline{c_{k}}=2\left(\overline{d_{1}}+\cdots+\overline{d_{g}}\right)=0\right\rangle .
$$

Observe that $\psi(K)=K$ and $K \subseteq \operatorname{ker} \theta_{i}$. If $\psi^{*}: H \rightarrow H, \theta_{i}^{*}: H \rightarrow C_{2}$ are the induced maps, then $\theta_{2}^{*}=\theta_{1}^{*} \psi^{*}$. Let $h=\overline{d_{1}}+\cdots+\overline{d_{g}}$. Note that $\psi^{*}$ permutes the $\overline{c_{i}}$ 's and $\psi^{*}(h)=h+\overline{c_{i_{1}}}+\cdots+\overline{c_{i_{2 s}}}$ for some $s \geq 0$ and $\left\{i_{1}, \ldots, i_{2 s}\right\} \subseteq\{1, \ldots, k\}$. Thus, $\theta_{2}^{*}(h)=$ $\theta_{1}^{*} \psi^{*}(h)=\theta_{1}^{*}(h)$. But $\theta_{i}^{*}(h)=\theta_{i}\left(d_{1} \cdots d_{g}\right)$, and so we have proved the necessity of condition (2). To prove the necessity of condition (3), suppose that $\theta_{1}\left(d_{1}\right)=\cdots=$ $\theta_{1}\left(d_{g}\right)=1$ and $\theta_{2}\left(d_{i}\right)=X$ for some $i \in\{1, \ldots, g\}$ and $\theta_{2}=\theta_{1} \psi$ for some $\psi \in \operatorname{Aut}(\Gamma)$. By condition (2) and proof of subcase 2(b), we may assume that $\theta_{2}\left(d_{1}\right)=\theta_{2}\left(d_{2}\right)=X$ and $\theta_{2}\left(d_{i}\right)=1$ for $i \geq 3$. For $i=1,2$, let $H_{i}=\operatorname{ker} \theta_{i} /\left[\operatorname{ker} \theta_{i}, \operatorname{ker} \theta_{i}\right]\left(H_{i}\right.$ is isomorphic to the first homology group with integral coefficients of the surface $\mathcal{H} / \operatorname{ker} \theta_{i}$ ) and let $p_{i}: \operatorname{ker} \theta_{i} \rightarrow H_{i}$ be the canonical projection. Let $\overline{e_{i}}=p_{1}\left(e_{i}\right)$ for $i=1, \ldots, k$, $\overline{c_{l}}=p_{1}\left(c_{k, 0} c_{l, 0}\right)$ for $l=1, \ldots, k-1, \overline{d_{j}}=p_{1}\left(d_{j}\right), \overline{f_{j}}=p_{1}\left(c_{k, 0} d_{j} c_{k, 0}\right)$ for $j=1, \ldots, g$. A presentation for ker $\theta_{1}$ may be obtained by the Reidemeister-Schreier procedure, then by taking abelianization we obtain that $H_{1}$ has a presentation (as a $\mathbb{Z}$-module) with generators $\overline{e_{i}}, \overline{c_{l}}, \overline{d_{j}}, \overline{f_{j}}$ satisfying the following two defining relations:

$$
\begin{aligned}
& \overline{e_{1}}+\cdots+\overline{e_{k}}=-2\left(\overline{d_{1}}+\cdots+\overline{d_{g}}\right), \\
& 2\left(\overline{d_{1}}+\cdots+\overline{d_{g}}-\left(\overline{f_{1}}+\cdots+\overline{f_{g}}\right)\right)=0 .
\end{aligned}
$$

Let $\widetilde{e}_{i}=p_{2}\left(e_{i}\right)$ for $i=1, \ldots, k, \widetilde{c}_{l}=p_{2}\left(c_{k, 0} c_{l, 0}\right)$ for $l=1, \ldots, k-1, \widetilde{d}_{j}=p_{2}\left(d_{j}\right), \widetilde{f}_{j}=$ $p_{2}\left(c_{k, 0} d_{j} c_{k, 0}\right)$ for $j=3, \ldots, g, a_{1}=p_{2}\left(d_{1} c_{k, 0}\right), b_{1}=p_{2}\left(c_{k, 0} d_{1}\right), a_{2}=p_{2}\left(d_{2} c_{k, 0}\right), b_{2}=$ $p_{2}\left(c_{k, 0} d_{2}\right)$. Then $H_{2}$ has a presentation with generators $\widetilde{e}_{i}, \widetilde{c}_{l}, \widetilde{d}_{j}, \widetilde{f}_{j}, a_{1}, b_{1}, a_{2}, b_{2}$ satisfying the following two defining relations:

$$
\begin{gathered}
\widetilde{e_{1}}+\cdots+\widetilde{e_{k}}=-\left(a_{1}+b_{1}+a_{2}+b_{2}+2\left(\widetilde{d_{3}}+\cdots+\widetilde{d_{g}}\right)\right), \\
2\left(\widetilde{d}_{3}+\cdots+\widetilde{d_{g}}-\left(\widetilde{f_{3}}+\cdots+\widetilde{f_{g}}\right)\right)=0 .
\end{gathered}
$$


Table 1. Groups $\mathrm{G}$ and $\Gamma$ for genus 4

\begin{tabular}{lcl}
\hline$G$ & Number & \multicolumn{1}{c}{$\Gamma$} \\
\hline$C_{2}$ & 1 & $(0,+,[2,2,2,2],\{(-)\})$ \\
& 2 & $(0,+,[2,2],\{(-),(-)\})$ \\
& 3 & $(0,+,[-],\{(-),(-),(-)\})$ \\
& 4 & $(1,-,[2,2,2,2],\{-\})$ \\
& 5 & $(1,-,[2,2],\{(-)\})$ \\
& 6 & $(1,-,[-],\{(-),(-)\})$ \\
& 7 & $(2,-,[2,2],\{-\})$ \\
& 8 & $(2,-,[-],\{(-)\})$ \\
& 9 & $(3,-,[-],\{-\})$ \\
$C_{3}$ & 10 & $(1,+,[-],\{(-)\})$ \\
$C_{2} \times C_{2}$ & & $(2,-,[3],\{-\})$ \\
& 1 & $(0,+,[2,2,2],\{(-)\})$ \\
& 2 & $(0,+,[2,2],\{(2,2)\})$ \\
& 3 & $(0,+,[2],\{(2,2,2,2)\})$ \\
& 4 & $(0,+,[-],\{(2,2,2,2,2,2)\})$ \\
& 5 & $(1,-,[2,2,2],\{-\})$ \\
$D_{3}$ & 6 & $(1,-,[2],\{(-)\})$ \\
& 7 & $(0,+,[2],\{(-)(-)\})$ \\
& & $(0,+,[2,2],\{(3)\})$ \\
& & $(0,+,[-],\{(-),(3)\})$ \\
& & $(1,-,[-],\{(3)\})$ \\
\hline
\end{tabular}

Consider the groups $H_{1}^{\prime}=H_{1} \otimes C_{2}, H_{2}^{\prime}=H_{2} \otimes C_{2}$ ( $H_{i}^{\prime}$ is isomorphic to the first homology group of $\mathcal{H} / \operatorname{ker} \theta_{i}$ with coefficients in $C_{2}$ ). For every $a \in H_{i}$, we denote by $[a]$ the element $a \otimes X \in H_{i}^{\prime}$. Let $h_{1}=\left[\overline{e_{1}}+\cdots+\overline{e_{k}}\right], h_{2}=\left[\widetilde{e_{1}}+\cdots+\widetilde{e_{k}}\right]$. Note that $h_{1}=0$ and $h_{2} \neq 0$. On the other hand, we claim that $\psi^{*}\left(h_{2}\right)=h_{1}$, where $\psi^{*}: H_{2}^{\prime} \rightarrow H_{1}^{\prime}$ is the isomorphism induced by $\psi$. To see this, note that for every $i, \psi\left(e_{i}\right)=y e_{j}^{ \pm 1} y^{-1}$ for some $j$ and some $y \in \Gamma$. If $y \in \operatorname{ker} \theta_{1}$ then $\psi^{*}\left[\tilde{e}_{i}\right]=\left[\overline{e_{j}}\right]$, and if $y \notin \operatorname{ker} \theta_{1}$ then $y c_{j, 0} \in \operatorname{ker} \theta_{1}$ and since $\psi\left(e_{i}\right)=y c_{j, 0} e_{j}^{ \pm 1}\left(y c_{j, 0}\right)^{-1}$, we also have $\psi^{*}\left[\widetilde{e}_{i}\right]=\left[\overline{e_{j}}\right]$. Thus, $\psi^{*}\left(h_{2}\right)=h_{1}$, which is a contradiction proving that $\theta_{1}$ and $\theta_{2}$ are not topologically conjugate and the condition (3) is necessary.

4. Surfaces of topological genus 4. As seen in Section 2.2, we need only to study the cyclic automorphism groups $C_{p}$ for $p$ prime. From [6] for $g=4$, the only such primes $p$ are 2 and 3. According to this we are going to consider the groups $C_{2}, C_{3}$, $C_{2} \times C_{2}$ and $D_{3}$. For each one of these groups, say $G$, we take from [9] the list of groups $\Gamma$ such that there exists an epimorphism $\theta: \Gamma \rightarrow G$ with $\operatorname{ker}(\theta)=\Gamma_{4}$. We give this list in Table 1.

First we consider the 10 signatures of $\Gamma$ corresponding to group $C_{2}$. We are going to define all epimorphisms $\theta: \Gamma \rightarrow C_{2}$ with kernel $\Gamma_{4}$, and to determine by using Theorem 2 whether two epimorphisms from the same NEC group are topologically conjugate.

Signature 1: $(0,+,[2,2,2,2],\{(-)\})$.

The unique epimorphism is given by

$$
\theta:\left(x_{1}, x_{2}, x_{3}, x_{4}, e_{1}, c_{1,0}\right) \rightarrow(X, X, X, X, 1, X)
$$

Signature 2: $(0,+,[2,2],\{(-),(-)\})$. 
There are two possible epimorphisms:

$$
\begin{aligned}
& \theta_{1}:\left(x_{1}, x_{2}, e_{1}, e_{2}, c_{1,0}, c_{2,0}\right) \rightarrow(X, X, 1,1, X, X), \\
& \theta_{2}:\left(x_{1}, x_{2}, e_{1}, e_{2}, c_{1,0}, c_{2,0}\right) \rightarrow(X, X, X, X, X, X) .
\end{aligned}
$$

By Theorem 2, the epimorphisms $\theta_{1}$ and $\theta_{2}$ are non-conjugate because $n_{1}=0$ and $n_{2}=2$. We call them cases 2.1 and 2.2.

Signature 3: $(0,+,[-],\{(-),(-),(-)\})$.

The unique epimorphism is given by

$$
\theta:\left(e_{1}, e_{2}, e_{3}, c_{1,0}, c_{2,0}, c_{3,0}\right) \rightarrow(X, X, 1, X, X, X) .
$$

Signature 4: $(1,-,[2,2,2,2],\{-\})$.

The epimorphism is given by

$$
\theta:\left(d_{1}, x_{1}, x_{2}, x_{3}, x_{4}\right) \rightarrow(1 \text { or } X, X, X, X, X),
$$

where the image of $d_{1}$ is irrelevant according to Theorem 2 since $r \neq 0$.

Signature 5: $(1,-,[2,2],\{(-)\})$.

The epimorphism is given by

$$
\theta:\left(d_{1}, x_{1}, x_{2}, e_{1}, c_{1,0}\right) \rightarrow(1 \text { or } X, X, X, 1, X),
$$

again the image of $d_{1}$ is irrelevant.

Signature 6: $(1,-,[-],\{(-),(-)\})$.

There are two kinds of epimorphisms given by

$$
\begin{aligned}
& \theta_{1}:\left(d_{1}, e_{1}, e_{2}, c_{1,0}, c_{2,0}\right) \rightarrow(1 \text { or } X, X, X, X, X), \\
& \theta_{2}:\left(d_{1}, e_{1}, e_{2}, c_{1,0}, c_{2,0}\right) \rightarrow(1,1,1, X, X) .
\end{aligned}
$$

The choice of $\theta_{1}\left(d_{1}\right)$ is irrelevant because $n_{1} \neq 0$. On the other hand, $\theta_{2}$ is not topologically conjugate to $\theta_{1}$ because $n_{1} \neq n_{2}$. We denote them as cases 6.1 and 6.2.

Signature 7: $(2,-,[2,2],\{-\})$.

The epimorphism is given by

$$
\theta:\left(d_{1}, d_{2}, x_{1}, x_{2}\right) \rightarrow(1 \text { or } X, 1 \text { or } X, X, X),
$$

and as above the choice for the images of $d_{1}$ and $d_{2}$ are irrelevant.

Signature 8: $(2,-,[-],\{(-)\})$.

The epimorphism is given by

$$
\theta:\left(d_{1}, d_{2}, e_{1}, c_{1,0}\right) \rightarrow(1,1 \text { or } X, 1, X),
$$

where the choice for the image of $d_{2}$ is irrelevant.

Signature 9: $(3,-,[-],\{-\})$.

There are three possible epimorphisms given by

$$
\begin{aligned}
& \theta_{1}:\left(d_{1}, d_{2}, d_{3}\right) \rightarrow(X, 1,1), \\
& \theta_{2}:\left(d_{1}, d_{2}, d_{3}\right) \rightarrow(X, X, X), \\
& \theta_{3}:\left(d_{1}, d_{2}, d_{3}\right) \rightarrow(X, 1, X) .
\end{aligned}
$$


We can see that $\theta_{1}\left(d_{1} d_{2} d_{3}\right)=\theta_{2}\left(d_{1} d_{2} d_{3}\right)=X$, while $\theta_{3}\left(d_{1} d_{2} d_{3}\right)=1$. Hence, by Theorem 2 , keeping in mind that $r=k=0$, there are two kinds of epimorphisms whose representatives are $\theta_{1}$ and $\theta_{3}$. We denote them as cases 9.1 and 9.2.

Signature 10: $(1,+,[-],\{(-)\})$.

The epimorphism is given by

$$
\theta:\left(a_{1}, b_{1}, e_{1}, c_{1,0}\right) \rightarrow(X, 1 \text { or } X, 1, X),
$$

where again the choice for the image of $b_{1}$ is irrelevant.

So we have 13 different cases denoted by $1,2.1,2.2,3,4,5,6.1,6.2,7,8,9.1,9.2$ and 10. For each of these there exists an epimorphism $\theta: \Gamma \rightarrow C_{2}$ with $\operatorname{ker}(\theta)=\Gamma_{4}$. According to the construction at the end of the Section 2.2, we are going to look for epimorphisms $\theta^{*}: \Gamma^{*} \rightarrow C_{2} \times C_{2}$ which connect any pair of epimorphisms.

We have in Table 1 the possible signatures for $\Gamma^{*}$. We call $\Gamma_{i}^{*}$ the NEC group with signature labeled $i$ in Table 1 . We shall run through these signatures until we connect the 13 cases. We exhibit the epimorphism $\theta^{*}$ in each case and distinguish carefully the situations 2.1 and 2.2, 6.1 and 6.2 and 9.1 and 9.2.

We denote the generators of $\Gamma^{*}$ with the corresponding starred letters. The generators of $C_{2} \times C_{2}$ are denoted by $X$ and $Y$.

First we take the group $\Gamma_{1}^{*}$ with signature $(0,+,[2,2,2],\{(-)\})$. Define the epimorphism $\theta^{*}: \Gamma_{1}^{*} \rightarrow C_{2} \times C_{2}$ by

$$
\left(x_{1}^{*}, x_{2}^{*}, x_{3}^{*}, e_{1}^{*}, c_{1,0}^{*}\right) \rightarrow(X, X, Y, Y, X) .
$$

Then $\theta^{*^{-1}}(\langle X\rangle)$ is an NEC group with signature $1, \theta^{*^{-1}}(\langle Y\rangle)$ is an NEC group with signature 7 and $\theta^{*^{-1}}(\langle X Y\rangle)$ is an NEC group with signature 9 . For the last one we must check whether we are in case 9.1 or 9.2. The generators of group $\theta^{*^{-1}}(\langle X Y\rangle)$ with signature $(3,-,[-],\{-\})$ can be expressed in terms of the generators of $\Gamma_{1}^{*}$ as

$$
\begin{aligned}
& d_{1}=x_{1}^{*} c_{1,0}^{*}, \\
& d_{2}=c_{1,0}^{*} x_{2}^{*}, \\
& d_{3}=x_{2}^{*} c_{1,0}^{*} x_{3}^{*} x_{2}^{*} .
\end{aligned}
$$

Hence, $\theta^{*}\left(d_{1}\right)=1, \theta^{*}\left(d_{2}\right)=1$ and $\theta^{*}\left(d_{3}\right)=X Y$. So this is case 9.1. As a consequence, cases 1,7 and 9.1 are connected,

$$
1-7-9.1
$$

Now we deal with the group $\Gamma_{2}^{*}$ with signature $(0,+,[2,2],\{(2,2)\})$. Define the epimorphism $\theta^{*}: \Gamma_{2}^{*} \rightarrow C_{2} \times C_{2}$ by

$$
\left(x_{1}^{*}, x_{2}^{*}, e_{1}^{*}, c_{1,0}^{*}, c_{1,1}^{*}, c_{1,2}^{*}\right) \rightarrow(X, Y, X Y, X, Y, X) .
$$

Then $\theta^{*^{-1}}(\langle X\rangle)$ is an NEC group with signature 5, and $\theta^{*^{-1}}(\langle X Y\rangle)$ is an NEC group with signature 7 . Hence, case 5 is also connected with the previous list,

$$
1-7-9.1-5
$$

We now define a new epimorphism from the same group $\Gamma_{2}^{*}$ by

$$
\left(x_{1}^{*}, x_{2}^{*}, e_{1}^{*}, c_{1,0}^{*}, c_{1,1}^{*}, c_{1,2}^{*}\right) \rightarrow(X, X, 1, X, Y, X) .
$$


For this epimorphism we have that $\theta^{*^{-1}}(\langle X\rangle)$ is an NEC group with signature 1, and $\theta^{*^{-1}}(\langle Y\rangle)$ is an NEC group with signature 8 . Case 8 is connected with the previous ones,

$$
1-7-9.1-5-8
$$

Let $\Gamma_{3}^{*}$ be an NEC group with signature $\left(0,+,[2],\{(2,2,2,2\})\right.$. Define $\theta^{*}: \Gamma_{3}^{*} \rightarrow$ $C_{2} \times C_{2}$ by

$$
\left(x_{1}^{*}, e_{1}^{*}, c_{1,0}^{*}, c_{1,1}^{*}, c_{1,2}^{*}, c_{1,3}^{*}, c_{1,4}^{*}\right) \rightarrow(X, X, X, Y, X, Y, X)
$$

Then $\theta^{*^{-1}}(\langle X\rangle)$ is an NEC group with signature 2, $\theta^{*^{-1}}(\langle Y\rangle)$ is an NEC group with signature 6 and $\theta^{*^{-1}}(\langle X Y\rangle)$ is an NEC group with signature 4 . We must distinguish which is the respective case for signatures 2 and 6 .

We express the generators of $\theta^{*^{-1}}(\langle X\rangle)$ in terms of the generators of $\Gamma_{3}^{*}$ as

$$
\begin{aligned}
x_{1} & =x_{1}^{*}, \\
x_{2} & =c_{1,3}^{*} x_{1}^{*} c_{1,3}^{*}, \\
e_{1} & =c_{1,3}^{*} c_{1,1}^{*}, \\
e_{2} & =c_{1,1}^{*} x_{1}^{*} c_{1,3}^{*} x_{1}^{*}, \\
c_{1,0} & =c_{1,2}^{*}, \\
c_{2,0} & =c_{1,0}^{*} .
\end{aligned}
$$

Then $\theta^{*}\left(e_{1}\right)=1$ and $\theta^{*}\left(e_{2}\right)=1$. Hence, this is case 2.1 .

We now deal with $\theta^{*^{-1}}(\langle Y\rangle)$. Its generators can be expressed as

$$
\begin{aligned}
d_{1} & =e_{1}^{*} c_{1,0}^{*}, \\
e_{1} & =c_{1,0}^{*} c_{1,2}^{*}, \\
e_{2} & =c_{1,2}^{*} c_{1,4}^{*}, \\
c_{1,0} & =c_{1,1}^{*}, \\
c_{2,0} & =c_{1,3}^{*} .
\end{aligned}
$$

Then $\theta^{*}\left(e_{1}\right)=1, \theta^{*}\left(e_{2}\right)=1$ and so this is case 6.2 . So we have seen that cases $2.1,4$ and 6.2 are connected. We have up to now

$$
1-7-9.1-5-8
$$

and

$$
2.1-4-6.2
$$

We now define a new epimorphism from the same group $\Gamma^{*}$ by

$$
\left(x_{1}^{*}, e_{1}^{*}, c_{1,0}^{*}, c_{1,1}^{*}, c_{1,2}^{*}, c_{1,3}^{*}, c_{1,4}^{*}\right) \rightarrow(X, X, X, Y, X Y, Y, X) .
$$

Then $\theta^{*^{-1}}(\langle X\rangle)$ has signature 1 , and $\theta^{*^{-1}}(\langle Y\rangle)$ has signature 6 . We now check whether this is case 6.1 or 6.2 . Since $\theta^{*}\left(e_{1}\right)=\theta^{*}\left(e_{2}\right)=Y$, this is case 6.1 . Hence, this case is 
connected with case 1 ,

$$
1-7-9.1-5-8-6.1
$$

Take now $\Gamma_{4}^{*}$ with signature $(0,+,[-],\{(2,2,2,2,2,2)\})$. Define $\theta^{*}: \Gamma_{4}^{*} \rightarrow C_{2} \times$ $C_{2}$ by

$$
\left(c_{1,0}^{*}, c_{1,1}^{*}, c_{1,2}^{*}, c_{1,3}^{*}, c_{1,4}^{*}, c_{1,5}^{*}, c_{1,6}^{*}\right) \rightarrow(X, X Y, X, Y, X, Y, X) .
$$

Then $\theta^{*^{-1}}(\langle X\rangle)$ has signature 3 , and $\theta^{*^{-1}}(\langle X Y\rangle)$ has signature 1 so that case 3 is connected with 1 ,

$$
1-7-9.1-5-8-6.1-3
$$

We now define another epimorphism $\theta^{*}: \Gamma_{4}^{*} \rightarrow C_{2} \times C_{2}$ by

$$
\left(c_{1,0}^{*}, c_{1,1}^{*}, c_{1,2}^{*}, c_{1,3}^{*}, c_{1,4}^{*}, c_{1,5}^{*}, c_{1,6}^{*}\right) \rightarrow(X, X Y, X, Y, X Y, Y, X) .
$$

Then both $\theta^{*^{-1}}(\langle Y\rangle)$ and $\theta^{*^{-1}}(\langle X Y\rangle)$ have signature 2. Consider first $\theta^{*^{-1}}(\langle Y\rangle)$. Its generators can be expressed by

$$
\begin{aligned}
x_{1} & =c_{1,0}^{*} c_{1,1}^{*}, \\
x_{2} & =c_{1,1}^{*} c_{1,2}^{*}, \\
e_{1} & =c_{1,2}^{*} c_{1,4}^{*}, \\
e_{2} & =c_{1,4}^{*} c_{1,6}^{*}, \\
c_{1,0} & =c_{1,3}^{*}, \\
c_{2,0} & =c_{1,5}^{*} .
\end{aligned}
$$

Then $\theta^{*}\left(e_{1}\right)=\theta^{*}\left(e_{2}\right)=Y$ and so this is case 2.2.

Take now $\theta^{*^{-1}}(\langle X Y\rangle)$. Its generators are expressed by

$$
\begin{aligned}
x_{1} & =c_{1,5}^{*} c_{1,3}^{*} c_{1,2}^{*} c_{1,5}^{*}, \\
x_{2} & =c_{1,5}^{*} c_{1,6}^{*}, \\
e_{1} & =c_{1,0}^{*} c_{1,2}^{*}, \\
e_{2} & =c_{1,3}^{*} c_{1,5}^{*}, \\
c_{1,0} & =c_{1,1}^{*}, \\
c_{2,0} & =c_{1,4}^{*} .
\end{aligned}
$$

Hence, $\theta^{*}\left(e_{1}\right)=\theta^{*}\left(e_{2}\right)=1$, and this is case 2.1 so that both cases are connected,

$$
2.1-4-6.2-2.2
$$

Now we have the group $\Gamma_{7}^{*}$ with signature $(0,+,[2],\{(-),(-)\})$ and define $\theta^{*}$ : $\Gamma_{7}^{*} \rightarrow C_{2} \times C_{2}$ by

$$
\left(x_{1}^{*}, e_{1}^{*}, e_{2}^{*}, c_{1,0}^{*}, c_{2,0}^{*}\right) \rightarrow(Y, 1, Y, Y, X) .
$$


Then $\theta^{*^{-1}}(\langle X\rangle)$ has signature $10, \theta^{*^{-1}}(\langle Y\rangle)$ has signature 2 and finally $\theta^{*^{-1}}(\langle X Y\rangle)$ has signature 9. $\Gamma_{7}^{*}$ by

We are going to express the generators of $\theta^{*^{-1}}(\langle Y\rangle)$ in terms of the generators of

$$
\begin{aligned}
x_{1} & =x_{1}^{*}, \\
x_{2} & =c_{2,0}^{*} x_{1}^{*} c_{2,0}^{*}, \\
e_{1} & =c_{2,0}^{*} e_{1}^{*} c_{2,0}^{*}, \\
e_{2} & =e_{1}^{*-1}, \\
c_{1,0} & =c_{2,0}^{*}, \\
c_{2,0} & =c_{1,0}^{*} .
\end{aligned}
$$

Hence, $\theta^{*}\left(e_{1}\right)=\theta^{*}\left(e_{2}\right)=1$, so this is case 2.1 .

We now deal with $\theta^{*^{-1}}(\langle X Y\rangle)$. Its generators can be expressed as

$$
\begin{aligned}
& d_{1}=x_{1}^{*} c_{1,0}^{*}, \\
& d_{2}=c_{1,0}^{*} x_{1}^{*} e_{1}^{*}, \\
& d_{3}=e_{2}^{*} c_{2,0}^{*} .
\end{aligned}
$$

Then $\theta^{*}\left(d_{1}\right)=\theta^{*}\left(d_{2}\right)=1$ and $\theta^{*}\left(d_{3}\right)=X Y$. So this is case 9.1 . Thus, cases 9.1 and 10 are connected with 2.1 ,

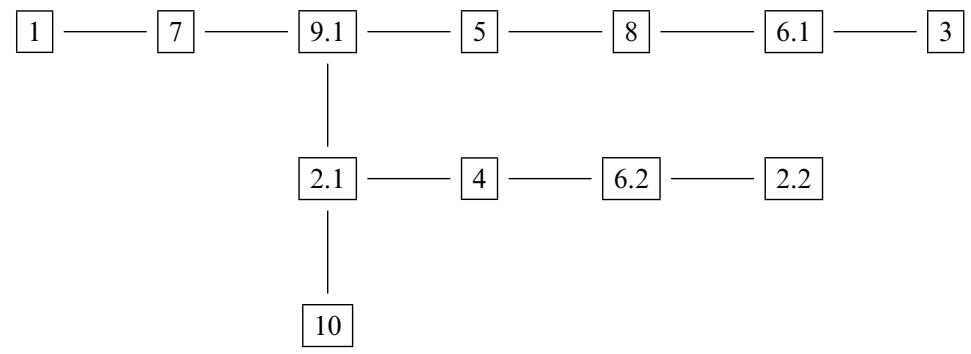

Up to now all cases are connected except for 9.2. We are dealing with this last case. We define a new epimorphism $\theta^{*}: \Gamma_{7}^{*} \rightarrow C_{2} \times C_{2}$ by

$$
\left(x_{1}, e_{1}, e_{2}, c_{1,0}, c_{2,0}\right) \rightarrow(Y, X Y, X, Y, Y) .
$$

Then $\theta^{*^{-1}}(\langle Y\rangle)$ has signature 2 and $\theta^{*^{-1}}(\langle X Y\rangle)$ has signature 9 . We must decide if we have the case 9.1 or 9.2 . The generators of the group $\theta^{*^{-1}}(\langle X Y\rangle)$ can be expressed as above. Then $\theta^{*}\left(d_{1}\right)=1, \theta^{*}\left(d_{2}\right)=\theta^{*}\left(d_{3}\right)=X Y$, and so this is case 9.2. Hence, case 9.2 is connected with one of the cases of signature 2 and we are done. We have finished to check that of all cases appearing for the prime $p=2$ are connected.

The following step deals with the other prime $p=3$. According to Table 1, the unique signature is $(2,-,[3],\{-\})$. We denote by $\Gamma$ a group with this signature. We are going to see that there are two epimorphisms $\theta: \Gamma \rightarrow C_{3}$ with kernel $\Gamma_{4}$ and check that they are conjugate by Theorem 1. Call $X$ the generator of $C_{3}$. The epimorphisms 
Table 2. Groups $\mathrm{G}$ and $\Gamma$ for genus 5

\begin{tabular}{lll}
\hline$G$ & Number & \multicolumn{1}{c}{$\Gamma$} \\
\hline$C_{2}$ & 1 & $(0,+,[2,2,2,2,2],\{(-)\})$ \\
& 2 & $(0,+,[2,2,2],\{(-),(-)\})$ \\
& 3 & $(0,+,[2],\{(-),(-),(-)\})$ \\
& 4 & $(1,-,[2,2,2],\{(-)\})$ \\
& 5 & $(1,-,[2],\{(-),(-)\})$ \\
& 6 & $(2,-,[2],\{(-)\})$ \\
$C_{3}$ & 7 & $(1,+,[2],\{(-)\})$ \\
& 1 & $(1,-,[3,3,3],\{-\})$ \\
$C_{2} \times C_{2}$ & 2 & $(3,-,[-],\{-\})$ \\
& 1 & $(0,+,[2,2],\{(2,2,2)\})$ \\
& 2 & $(0,+,[2],\{(2,2,2,2,2)\})$ \\
& 3 & $(0,+,[-],\{(2,2,2,2,2,2,2)\})$ \\
$C_{5}$ & 4 & $(0,+,[-],\{(2,2,2),(-)\})$ \\
$D_{3}$ & 5 & $(1,-,[-],\{(2,2,2)\})$ \\
& 1 & $(1,-,[5,5],\{-\})$ \\
& 2 & $(0,+,[2,3],\{(3)\})$ \\
& 3 & $(0,+,[2],\{(3,3,3)\})$ \\
& 4 & $(0,+,[2,2,2],\{(-)\})$ \\
$D_{5}$ & 5 & $(1,-,[2],\{(-),(-)\})$ \\
& 1 & $(0,+,[2,5],\{(-)\})$ \\
& 2 & $(0,+,[2],\{(5,5)\})$ \\
\hline
\end{tabular}

are defined by

$$
\left(d_{1}, d_{2}, x_{1}\right) \rightarrow(X, 1, X) \quad \text { or } \quad\left(d_{1}, d_{2}, x_{1}\right) \rightarrow\left(X^{2}, X^{2}, X\right)
$$

and because the images of $x_{1}$ are the same, both epimorphisms are conjugate.

By [9], the action of $\Gamma$ extends to a group $\Gamma^{*}$ such that there exists an epimorphism $\theta^{*}: \Gamma^{*} \rightarrow D_{3}$ with kernel $\Gamma_{4}$. Let $X, Y \in D_{3}$ have orders 2 and 3 . Then $\theta^{*^{-1}}(\langle X\rangle)$ is one of the above studied groups corresponding to $C_{2}$, while $\theta^{*^{-1}}(\langle Y\rangle)$ is $\Gamma$. So the unique case corresponding to $C_{3}$ is connected to one of the cases of $C_{2}$, and then to all of them. We have finished to prove the following.

THEOREM 3. The space $B_{4}$ is connected.

5. Surfaces of topological genus 5. We are going to consider the groups $C_{p}$ for $p$ prime acting on surfaces of genus $g=5$. From [6], the values of $p$ are 2, 3 or 5. Hence, we consider the groups $C_{2}, C_{3}, C_{5}, C_{2} \times C_{2}, D_{3}$ and $D_{5}$. For each of these groups, say $G$, the list of groups $\Gamma$ such that there exists an epimorphism $\theta: \Gamma \rightarrow G$ with $\operatorname{ker}(\theta)=\Gamma_{5}$ is taken from [9] and collected in Table 2.

We are going to consider the seven signatures of $\Gamma$ corresponding to the group $C_{2}$. We determine all epimorphisms $\theta: \Gamma \rightarrow C_{2}$ with kernel $\Gamma_{5}$ which are topologically non-conjugate. As in the previous section, we call $X$ the generator of $C_{2}$.

Signature 1: $(0,+,[2,2,2,2,2],\{(-)\})$.

The unique epimorphism is given by

$$
\theta:\left(x_{1}, x_{2}, x_{3}, x_{4}, x_{5}, e_{1}, c_{1,0}\right) \rightarrow(X, X, X, X, X, X, X) .
$$

Signature 2: $(0,+,[2,2,2],\{(-),(-)\})$. 
The unique epimorphism is given by

$$
\theta:\left(x_{1}, x_{2}, x_{3}, e_{1}, e_{2}, c_{1,0}, c_{2,0}\right) \rightarrow(X, X, X, X, 1, X, X) .
$$

Signature 3: $(0,+,[2],\{(-),(-),(-)\})$.

There are two possible epimorphisms given by

$$
\begin{aligned}
& \theta_{1}:\left(x_{1}, e_{1}, e_{2}, e_{3}, c_{1,0}, c_{2,0}, c_{3,0}\right) \rightarrow(X, X, X, X, X, X, X), \\
& \theta_{2}:\left(x_{1}, e_{1}, e_{2}, e_{3}, c_{1,0}, c_{2,0}, c_{3,0}\right) \rightarrow(X, X, 1,1, X, X, X) .
\end{aligned}
$$

By Theorem 2, the epimorphisms $\theta_{1}$ and $\theta_{2}$ are non-conjugate because $n_{1}=2$ and $n_{2}=0$. We call these cases 3.1 and 3.2.

Signature 4: $(1,-,[2,2,2],\{(-)\})$.

The unique epimorphism is given by

$$
\theta:\left(d_{1}, x_{1}, x_{2}, x_{3}, e_{1}, c_{1,0}\right) \rightarrow(1 \text { or } X, X, X, X, X, X) .
$$

The choice of the image of $d_{1}$ is irrelevant according to Theorem 2 because $r \neq 0$.

Signature 5: $(1,-,[2],\{(-),(-)\})$.

The unique epimorphism is given by

$$
\theta:\left(d_{1}, x_{1}, e_{1}, e_{2}, c_{1,0}, c_{2,0}\right) \rightarrow(1 \text { or } X, X, X, 1, X, X) .
$$

Again, the choice of the image of $d_{1}$ is irrelevant.

Signature 6: $(2,-,[2],\{(-)\})$.

The unique epimorphism is given by

$$
\theta:\left(d_{1}, d_{2}, x_{1}, e_{1}, c_{1,0}\right) \rightarrow(1 \text { or } X, 1 \text { or } X, X, X, X) .
$$

As in the previous cases, the choice of the images of $d_{1}$ and $d_{2}$ are irrelevant.

Signature 7: $(1,+,[2],\{(-)\})$.

The unique epimorphism is given by

$$
\theta:\left(a_{1}, b_{1}, x_{1}, e_{1}, c_{1,0}\right) \rightarrow(1 \text { or } X, 1 \text { or } X, X, X, X) .
$$

Again, the choice of the images of $a_{1}$ and $b_{1}$ are irrelevant.

Hence, we have eight different cases denoted by 1, 2, 3.1, 3.2, 4, 5, 6 and 7. For each of these there exists an epimorphism $\theta: \Gamma \rightarrow C_{2}$ with $\operatorname{ker}(\theta)=\Gamma_{5}$. We are going to look for epimorphisms $\theta^{*}: \Gamma^{*} \rightarrow C_{2} \times C_{2}$ which connect all epimorphisms $\theta$.

The possible signatures for $\Gamma^{*}$ appear in Table 2. We call $\Gamma_{i}^{*}$ the NEC group with signature labeled $i$ in Table 2 for the group $C_{2} \times C_{2}$. We denote the generators of $\Gamma^{*}$ with the corresponding starred letters, and call $X$ and $Y$ the generators of $C_{2} \times C_{2}$.

First we take the group $\Gamma_{1}^{*}$ with signature $(0,+,[2,2],\{(2,2,2)\})$. Define an epimorphism $\theta^{*}: \Gamma_{1}^{*} \rightarrow C_{2} \times C_{2}$ by

$$
\left(x_{1}^{*}, x_{2}^{*}, e_{1}^{*}, c_{1,0}^{*}, c_{1,1}^{*}, c_{1,2}^{*}, c_{1,3}^{*}\right) \rightarrow(X, X, 1, X, Y, X Y, X) .
$$

Then $\theta^{*^{-1}}(\langle X\rangle)$ is an NEC group with signature 1, and $\theta^{*^{-1}}(\langle Y\rangle)$ is an NEC group with signature 6 . Hence, cases 1 and 6 are connected,

$$
1-6
$$


We define a different epimorphism from the same group $\Gamma_{1}^{*}$ by

$$
\left(x_{1}^{*}, x_{2}^{*}, e_{1}^{*}, c_{1,0}^{*}, c_{1,1}^{*}, c_{1,2}^{*}, c_{1,3}^{*}\right) \rightarrow(X, Y, X Y, X, Y, X Y, X) \text {. }
$$

For this epimorphism we have that $\theta^{*^{-1}}(\langle X\rangle)$ is an NEC group with signature 4, and $\theta^{*^{-1}}(\langle X Y\rangle)$ is an NEC group with signature 6 . Hence, case 4 is connected with the previous ones,

$$
1-6-4
$$

Now we take $\Gamma_{2}^{*}$ as an NEC group with signature $(0,+,[2],\{(2,2,2,2,2)\})$. Define $\theta^{*}: \Gamma_{2} \rightarrow C_{2} \times C_{2}$ by

$$
\left(x_{1}^{*}, e_{1}^{*}, c_{1,0}^{*}, c_{1,1}^{*}, c_{1,2}^{*}, c_{1,3}^{*}, c_{1,4}^{*}, c_{1,5}^{*}\right) \rightarrow(X, X, Y, X, Y, X Y, X, Y) .
$$

Then $\theta^{*^{-1}}(\langle X\rangle)$ is an NEC group with signature 2, $\theta^{*^{-1}}(\langle Y\rangle)$ is an NEC group with signature 5 and $\theta^{*^{-1}}(\langle X Y\rangle)$ is an NEC group with signature 4 . Hence, cases 2 and 5 are connected with the three previous ones,

$$
1-6-4-2-5
$$

Now, let $\Gamma_{3}^{*}$ be an NEC group with signature $(0,+,[-],\{(2,2,2,2,2,2,2)\})$. Define an epimorphism $\theta^{*}$ from this group onto $C_{2} \times C_{2}$ by

$$
\left(c_{1,0}^{*}, c_{1,1}^{*}, c_{1,2}^{*}, c_{1,3}^{*}, c_{1,4}^{*}, c_{1,5}^{*}, c_{1,6}^{*}, c_{1,7}^{*}\right) \rightarrow(X, X Y, X, X Y, Y, X, Y, X) .
$$

Then $\theta^{*^{-1}}(\langle X\rangle)$ is an NEC group with signature 3, and $\theta^{*^{-1}}(\langle Y\rangle)$ is an NEC group with signature 2 . We must distinguish whether this is case 3.1 or 3.2 . We express the generators of $\theta^{*^{-1}}(\langle X\rangle)$ as follows:

$$
\begin{aligned}
x_{1} & =c_{1,3}^{*} c_{1,4}^{*}, \\
e_{1} & =c_{1,4}^{*} c_{1,6}^{*}, \\
e_{2} & =c_{1,6}^{*} c_{1,1}^{*}, \\
e_{3} & =c_{1,1}^{*} c_{1,3}^{*}, \\
c_{1,0} & =c_{1,5}^{*}, \\
c_{2,0} & =c_{1,7}^{*}, \\
c_{3,0} & =c_{1,2}^{*} .
\end{aligned}
$$

Then $\theta^{*}\left(e_{1}\right)=1, \theta^{*}\left(e_{2}\right)=X$ and $\theta^{*}\left(e_{3}\right)=1$. So this is case 3.2 and is connected with case 2 ,

$$
1-6-4-2-5-3.2
$$

We now consider an NEC group $\Gamma_{4}^{*}$ with signature $(0,+,[-],\{(2,2,2),(-)\})$. Define an epimorphism $\theta^{*}$ from this group onto $C_{2} \times C_{2}$ by

$$
\left(e_{1}^{*}, e_{2}^{*}, c_{1,0}^{*}, c_{1,1}^{*}, c_{1,2}^{*}, c_{1,3}^{*}, c_{2,0}^{*}\right) \rightarrow(X, X, X, X Y, Y, X, X) .
$$


Then $\theta^{*^{-1}}(\langle X\rangle)$ is an NEC group with signature 3, and $\theta^{*^{-1}}(\langle Y\rangle)$ is an NEC group with signature 7. We must distinguish whether the former is case 3.1 or 3.2. We express the generators of $\theta^{*^{-1}}(\langle X\rangle)$ as follows:

$$
\begin{aligned}
x_{1} & =c_{1,2}^{*} c_{1,1}^{*}, \\
e_{1} & =c_{1,1}^{*} e_{1}^{*} c_{1,2}^{*} e_{1}^{*^{-1}}, \\
e_{2} & =e_{2}^{*^{-1}}, \\
e_{3} & =c_{1,2}^{*} e_{2}^{*} c_{1,2}^{*}, \\
c_{1,0} & =c_{1,0}^{*}, \\
c_{2,0} & =c_{2,0}^{*}, \\
c_{3,0} & =c_{1,2}^{*} c_{2,0}^{*} c_{1,2}^{*} .
\end{aligned}
$$

Then $\theta^{*}\left(e_{1}\right)=\theta^{*}\left(e_{2}\right)=\theta^{*}\left(e_{3}\right)=X$. So this is case 3.1 and is connected with Case 7 ,

$$
7-3.1
$$

Take an NEC group $\Gamma_{5}^{*}$ with signature $(1,-,[-],\{(2,2,2)\})$. Define $\theta^{*}: \Gamma_{5}^{*} \rightarrow$ $C_{2} \times C_{2}$ by

$$
\left(d_{1}^{*}, e_{1}^{*}, c_{1,0}^{*}, c_{1,1}^{*}, c_{1,2}^{*}, c_{1,3}^{*}\right) \rightarrow(X Y, 1, X, Y, X Y, X)
$$

Then $\theta^{*^{-1}}(\langle X\rangle)$ is an NEC group with signature 7 and $\theta^{*^{-1}}(\langle X Y\rangle)$ is an NEC group with signature 6 . Hence, case 7 (and also case 3.1 ) is connected with the previous ones,

$$
1-6-4-2-5-3.2-7-3.1
$$

We have proven that all cases for prime $p=2$ are connected.

We now deal with the prime $p=3$. We denote by $X$ the generator of $C_{3}$. According to Table 2 we must consider two signatures.

Signature 1: $(1,-,[3,3,3],\{-\})$.

There are three possible epimorphisms given by

$$
\begin{aligned}
& \theta_{1}:\left(d_{1}, x_{1}, x_{2}, x_{3}\right) \rightarrow(1, X, X, X), \\
& \theta_{2}:\left(d_{1}, x_{1}, x_{2}, x_{3}\right) \rightarrow\left(X, X, X, X^{2}\right), \\
& \theta_{3}:\left(d_{1}, x_{1}, x_{2}, x_{3}\right) \rightarrow\left(X^{2}, X, X^{2}, X^{2}\right) .
\end{aligned}
$$

In order to apply Theorem 1, observe that the respective images of the elliptic elements are $\{X, X, X\},\left\{X, X, X^{2}\right\}$ and $\left\{X, X^{2}, X^{2}\right\}$. Since $X^{2}=X^{-1}$, the epimorphism $\theta_{1}$ is topologically conjugate to both $\theta_{2}$ and $\theta_{3}$, and so these are all equivalent.

Signature 2: $(3,-,[-],\{-\})$.

There are two possible epimorphisms given by

$$
\begin{aligned}
& \theta_{1}:\left(d_{1}, d_{2}, d_{3}\right) \rightarrow(X, X, X), \\
& \theta_{2}:\left(d_{1}, d_{2}, d_{3}\right) \rightarrow\left(1, X, X^{2}\right) .
\end{aligned}
$$

Since $r=0$, both of these are topologically conjugate by Theorem 1 . 
We are going to extend both actions to $D_{3}$, and so these two cases will be connected to those corresponding to $p=2$. For that we consider an NEC group $\Gamma_{2}^{*}$ with signature $(0,+,[2],\{(3,3,3)\})$, which is the second signature in Table 2 corresponding to group $D_{3}$. We denote by $X$ and $Y$ the generators of $D_{3}$ satisfying $X^{2}=Y^{2}=(X Y)^{3}=1$. We define an epimorphism $\theta^{*}: \Gamma_{2}^{*} \rightarrow D_{3}$ by

$$
\left(x_{1}^{*}, e_{1}^{*}, c_{1,0}^{*}, c_{1,1}^{*}, c_{1,2}^{*}, c_{1,3}^{*}\right) \rightarrow(Y, Y, X, Y, X, Y X Y) .
$$

Then $\theta^{*^{-1}}(\langle X Y\rangle)$ has signature 1 , and $\theta^{*^{-1}}(\langle X\rangle)$ is a group corresponding to $C_{2}$. So case 1 is connected with a case of $C_{2}$ and so with all of them.

We are now going to make the same with signature 2 . Call $\Gamma_{5}^{*}$ an NEC group with signature $(1,-,[2],\{(-)\})$, the fifth signature in Table 2 corresponding to $D_{3}$. Define $\theta^{*}: \Gamma_{5}^{*} \rightarrow D_{3}$ by

$$
\left(d_{1}^{*}, x_{1}^{*}, e_{1}^{*}, c_{1,0}^{*}\right) \rightarrow(X Y, X, Y, Y) .
$$

Then $\theta^{*^{-1}}(\langle X Y\rangle)$ has signature 2 and $\theta^{*^{-1}}(\langle X\rangle)$ is a group corresponding to $C_{2}$. So case 2 is connected with a case of $C_{2}$ and so with all of them.

Finally, we deal with the prime $p=5$. There exists a unique signature for group $C_{5}$ in Table 2, which is $(1,-,[5,5],\{-\})$. Call $\Gamma$ an NEC group with this signature. Applying Theorem 1 as in the previous case, we see that there are two kinds of topologically non-conjugate epimorphisms from $\Gamma$ onto $C_{5}$ represented by

$$
\begin{aligned}
& \theta_{1}:\left(d_{1}, x_{1}, x_{2}\right) \rightarrow\left(X^{4}, X, X\right), \\
& \theta_{2}:\left(d_{1}, x_{1}, x_{2}\right) \rightarrow\left(X, X, X^{2}\right),
\end{aligned}
$$

where $X$ denotes a generator of $C_{5}$. In order to keep the same notation, we call them cases 1.1 and 1.2. We are going to connect both cases to those of $C_{2}$ by extending the action to $D_{5}$. As usual we call $X$ and $Y$ the generators of $D_{5}$ satisfying $X^{2}=Y^{2}=$ $(X Y)^{5}=1$.

First, let $\Gamma_{1}^{*}$ be an NEC group with signature $(0,+,[2,5],\{(-)\})$, the first signature for $D_{5}$ in Table 2. Define $\theta^{*}: \Gamma_{1}^{*} \rightarrow D_{5}$ by

$$
\left(x_{1}^{*}, x_{2}^{*}, e_{1}^{*}, c_{1,0}^{*}\right) \rightarrow(X, X Y, Y, Y) .
$$

We consider the group $\theta^{*^{-1}}(\langle X Y\rangle)$. Its generators can be expressed in terms of the generators of $\Gamma_{1}^{*}$ by

$$
\begin{aligned}
& d_{1}=x_{1}^{*} c_{1,0}^{*}, \\
& x_{1}=c_{1,0}^{*} x_{2}^{*} c_{1,0}^{*}, \\
& x_{2}=x_{2}^{*} .
\end{aligned}
$$

Then $\theta^{*}\left(x_{1}\right)=Y X=(X Y)^{-1}$ and $\theta^{*}\left(x_{2}\right)=X Y$, hence by applying Theorem 1 , this epimorphism is topologically conjugate to $\theta_{1}$ and this is case 1.1 . Since $\theta^{*^{-1}}(\langle X\rangle)$ is an NEC group corresponding to $C_{2}$, case 1.1 is connected with those corresponding to $\mathrm{C}_{2}$. 
Take now $\Gamma_{2}^{*}$ an NEC group with signature $(0,+,[2],\{(5,5)\})$, the second signature for $D_{5}$ in Table 2. Define $\theta^{*}: \Gamma_{2}^{*} \rightarrow D_{5}$ by

$$
\left(x_{1}^{*}, e_{1}^{*}, c_{1,0}^{*}, c_{1,1}^{*}, c_{1,2}^{*}\right) \rightarrow(X Y X Y X, X Y X Y X, X, X Y X, Y) .
$$

We consider the group $\theta^{*^{-1}}(\langle X Y\rangle)$. Its generators can be expressed in terms of the generators of $\Gamma_{2}^{*}$ by

$$
\begin{aligned}
& d_{1}=x_{1}^{*} c_{1,0}^{*}, \\
& x_{1}=c_{1,0}^{*} c_{1,1}^{*}, \\
& x_{2}=c_{1,1}^{*} c_{1,2}^{*} .
\end{aligned}
$$

Then $\theta^{*}\left(x_{1}\right)=Y X=(X Y)^{-1}$ and $\theta^{*}\left(x_{2}\right)=(X Y)^{2}$. So this epimorphism is topologically conjugate to $\theta_{2}$, and this is case 1.2. Arguing as above, this case is connected to those corresponding to $C_{2}$.

We have finished to connect all possibilities for the primes $p=2,3$ and 5, and so we have proved the following.

THEOREM 4. The space $B_{5}$ is connected.

ACKNOWLEDGEMENTS. The authors wish to thank the referee for his/her careful reading and suggestions on additional references in order to enlarge the context of the paper. The first and third authors are partially supported by MTM2011-23092, the second author by UCM910444 and MTM2011-22435 and the fourth one by NN201 366436 and NCN 2012/05/B/ST1/02171.

\section{REFERENCES}

1. N. L. Alling and N. Greenleaf, Foundations of the theory of Klein surfaces, Lecture Notes in Mathematics, vol. 219 (Springer-Verlag, Berlin, Germany, 1971).

2. G. Bartolini, A. F. Costa and M. Izquierdo, On the connectivity of branch loci of moduli spaces, Ann. Acad. Sci. Fenn. Math. 38 (2013), 245-258.

3. G. Bartolini, A. F. Costa, M. Izquierdo and A. M. Porto, On the connectedness of the branch locus of the moduli space of Riemann surfaces, Rev. R. Acad. Cienc. Ser. A. Math. 104 (2010), 81-86.

4. G. Bartolini and M. Izquierdo, On the connectedness of the branch locus of the moduli space of Riemann surfaces of low genus, Proc. Am. Math. Soc. 140 (2012), 35-45.

5. S. A. Broughton, The equisymmetric stratification of the moduli space and the Krull dimension of mapping class groups, Topology Appl. 37 (1990), 101-113.

6. E. Bujalance, Cyclic groups of automorphisms of compact non-orientable Klein surfaces without boundary, Pac. J. Math. 109 (1983), 279-289.

7. E. Bujalance and A. F. Costa, Orientation reversing automorphisms of Riemann surfaces, Illinois J. Math. 38 (1994), 616-623.

8. E. Bujalance, A. F. Costa, S. Natanzon and D. Singerman, Involutions of compact Klein surfaces, Math. Z. 211 (1992), 461-478.

9. E. Bujalance, J. J. Etayo and E. Martínez, The full group of automorphisms of nonorientable unbordered Klein surfaces of topological genus 3, 4 and 5, Rev. Mat. Complut. 27 (2014), 305-326.

10. J. A. Bujalance, A. F. Costa and A. M. Porto, On the connectedness of the locus of real elliptic-hyperelliptic Riemann surfaces, Int. J. Math. 20 (2009), 1069-1080.

11. P. Buser, M. Seppälä and R. Silhol, Triangulations and moduli spaces of Riemann surfaces with group actions, Manuscr. Math. 88 (1995), 209-224. 
12. A. F. Costa and M. Izquierdo, On the connectedness of the locus of real Riemann surfaces, Ann. Acad. Fenn. Math. 27 (2002), 341-356.

13. A. F. Costa and M. Izquierdo, On the connectedness of the branch locus of the moduli space of Riemann surfaces of genus 4, Glasgow Math. J. 52 (2010), 401-408.

14. W. J. Harvey, On branch loci in Teichmüller space, Trans. Am. Math. Soc. 153 (1971), 387-399.

15. R. S. Kulkarni, Isolated points in the branch locus of the moduli space of compact Riemann surfaces, Ann. Acad. Fenn. Sci. Math. 16 (1991), 71-81.

16. A. M. Macbeath, The classification of non-Euclidean crystallographic groups, Can. J. Math. 19 (1967), 1192-1205.

17. A. M. Macbeath and D. Singerman, Spaces of subgroups and Teichmüller space, Proc. London Math. Soc. 31 (1975), 211-256.

18. S. M. Natanzon, Klein surfaces, Russ. Math. Surv. 45 (1990), 43-108.

19. R. Preston, Projective structures and fundamental domains on compact Klein surfaces, PhD Thesis (University of Texas, Austin, TX, 1975).

20. M. Seppälä, Moduli spaces of stable real algebraic curves, Ann. Sci. Éc. Norm. Sup. (4) 24 (1991), 519-544.

21. D. Singerman, Symmetries of Riemann surfaces with large automorphism group, Math. Ann. 210 (1974), 17-32.

22. H. C. Wilkie, On non-Euclidean crystallographic groups, Math. Z. 91 (1966), 87-102. 